\title{
Predictors of Work-ability over the Lifespan
}

\section{Trevor Archer* and Max Rapp-Ricciardi}

Department of Psychology, Gothenburg University, Sweden

"Work-ability" as a concept refers to a comprehensive notion for occupational health re-search and illness prevention. With the current tendencies for abolishment of early retire-ment combined with the increase of the statutory retirement age among Western Europe-an Communities, the number of self-employed/ older workers will most likely increase and that older workers with/without disabilities will be required to work longer.

The notion of work-ability provides a basic and reliable explanatory framework for the understanding of some elements of sustainable employability amongst a range of professionals. Thus, there is progressively an emerging reality, for example, among self-employed workers, whereby three aspects have been noted: i) the differences in selfrated health and work-ability of self-employed workers and employees, ii) whether self-employment is associated with better self-rated health and work ability across three years, than employment, and iii) the role of sociodemographic, health- and work-related characteristics (e.g., mental load, physical load, frequency of absenteeism, and autonomy) in these relationships [1]. Observations of employers' perceptions of employees presenting mental health problems remains an important buttressing the coping strategies and efforts of individuals within their work environments has been found to modulate work-ability. Individuals' proximal environmental surroundings appear to interact with the development and maturation of their brain whereby a critical aspect of this individual-environment interaction is defined by his/her socioeconomic status, through which one estimates access-to-material resources and social prestige levels. Previous characterizations of the relation between socioeconomic status and the brain have primarily focused upon earlier or later epochs of the individual's lifespan (i.e., childhood, adolescence, young adult, middle age, elder and older elder age). As defined by education level and attainment and occupational socioeconomic characteristics, socioeconomic status has been found to moderate those previously reported age-related differences in the individual's regional functional brain's network organization and whole-brain cortical structure and function [2]. Throughout and across the middle age years (35-64 years), lower socioeconomic status has been related to a reduced resting-state system segregation, which presents a measure of effective functional network organization. An analogous although less robust relationship exists between socioeconomic status and age with respect to brain anatomy features: Lower socioeconomic status has been associated with reduced cortical gray matter thickness during middle age. Conversely, younger and older adulthood do not exhibit consistent socioeconomic status-related difference in the brain measures. The SES-brain relationships persist after controlling for measures of physical and mental health, neurocognitive ability, and participant demographics.

Critically, an individual's childhood socioeconomic status seems not to account for the relationship between their current socioeconomic status and functional network organization thereby presenting ample evidence that socioeconomic status relates to the brain's functional network organization and anatomy across adult middle age, whereas the higher levels of socioeconomic status provide a protective factor against age-related brain decline thereby offering an essential relationship for the role of socioeconomic status in the provision of neurohealth and work-ability. It has been observed that higher scores in self-rated health and work-ability among the self-employed than employees. The status of being self-employed leads to an increase in work-ability across three years. The differences in work-ability may be considered small in many cases, and much ongoing research is currently focused upon the establishment of the role of self-employment as a potential facilitator for sustainable employment in the context of work-ability sustenance [3]. Work-ability, at older ages is accumulated over the lifespan, particularly through the prevailing health conditions during childhood and adolescence, ages at which working-life is initiated, educational level, and health conditions during the advancing years. Both mental health and somatic attributes mediate the efficacy of coping strategies among workers: Active coping strategies mediated between fewer incidents pertaining to self-reported physical limitations, higher levels of depressive symptoms, improved cognitive functioning and greater fatigue, on the one hand, and more physical limitations and limitations in working hours, on the other hand whereas passive coping played no mediating role and was associated with higher levels self-reported depressive symptoms only. Among patients/clients presenting chronic whiplash-associated disorders, neck-specific exercise, particularly with a behavioral approach, may have a more positive impact on healthrelated quality of life than physical activity prescription in chronic whiplash-associated disorders grades 2 and 3 [4]. Within the context of the unemployed work-ability emerges as emerging critical factors for health promotion, i.e. both women and men have been shown to exhibit a wide range of unhealthy/self-destructive behaviors, such as poor/unrestricted diet, daily smoking habit and risk level-usage of alcohol/abuse drugs. For purposes of health promotion, measures that enhancing a healthy lifestyle and sufficient maintenance workability among unemployed individuals, with particular reference to the importance of physical exercise facilities hold great promise.

A higher incidence of health-related problems among a very large population of lower and elder middle-aged individuals has been found to be associated with poorer work-ability although this negative effect stabilized from multi-health problems onwards whereby combinations of physical and mental health problem(s) were related more strongly to poorer work-ability than the presence of physical health problems only. The negative relation between physical health problems and work ability was partially suppressed by active coping, whereas the negative relation between the combination of physical and mental health problem(s) on work ability was explained partially by avoidant coping behavior [5]. The associations of social support networks with

${ }^{*}$ Corresponding author: Trevor Archer, Department of Psychology, Gothenburg University, Sweden, Tel: + 4631786 4694; E-mail: trevor.archer@psy.gu.se

Received: December 05, 2018; Accepted: December 13, 2018; Published: December 20, 2018

Citation: Archer T, Rapp-Ricciardi M (2018) Predictors of Work-ability over the Lifespan. Clin Exp Psychol 4: 203. doi: 10.4172/2471-2701.1000203

Copyright: @ 2018 Archer T, et al. This is an open-access article distributed under the terms of the Creative Commons Attribution License, which permits unrestricted use, distribution, and reproduction in any medium, provided the original author and source are credited. 
mental health parameters, subjective work-ability and psychological consultation present essential ingredients. Following adjustment for socio-demographic data, work-related characteristics and personality factors, persons with low social support compared to high social support presented poorer mental health and a lower level of work ability, according to the work-ability index with Low social support not being associated with increased odds of consulting a psychologist [6]. It has been observed that employees' perceptions of work-ability included experiences of diffuse and unexpressed signs of the onset of MHP and frustration among employers and work-mates, which was difficult to verbalize [7]. A strong and durable relationship between nurses work ability and Work-related Quality of Life was obtained that included mental resources, number of current diseases, and work ability in relation to the pervading job demands among a large sample of Iranian nurses [8]. The early retirement induced by chronic solventexposure encephalopathy due to the occupational hazard, an obstacle to work-ability and sustainability, is characterized by neurocognitive impairments, mood and psychiatric symptoms and dementia, as well as memory and concentration-attention symptoms, work-ability and solvent-related and non-occupational factors on work-ability, in actively-working solvent-exposed populations. Memory and concentration-attention symptoms were linked with higher solvent exposure whereas influences upon self-experienced work-ability were of a relatively weak order, confirming outcomes based upon the improved occupational hygiene and reduced solvent exposure levels pursued among industrialized countries implying that the effects may be stronger in high-level exposure environments [9].

Under occupational conditions defined by continual, chronic, high, unpredictable stress involving specific tasks by highly trained professionals, the relationships between work-ability and health may be difficult and complex, with problems emerging under laboratory conditions. In considerations of work-related and individual factors that remain separately- and jointly-related to psychological-somatic work ability and sustained employment intentions via late job mobility both personal and physiologic attributes have been examined. Individuals, tasked with maintaining peak level of performance under stressful and often unpredictable conditions, therewith wrestling chronically with stressful occupations, such as law enforcement, general practitioners and military personnel, are expected to operate within high stakes environments that may be both physically and emotionally simultaneously demanding will be exerting invariably high levels of cognitive-emotional control in order to sustain attentionto-task and suppressive measures upon task-irrelevant behaviors. In a study applying emotional stress induced by threat of unpredictable electric shock that mitigated the effects of physical stress upon response inhibition, during task-performance involving an auditory Go/NoGo task under safe versus threat conditions while cycling at high intensity, i.e. $84 \%$ max heart rate for $50 \mathrm{~min}$, the participants were instructed that they would receive mild electric shocks designed to be unpredictable and unrelated to task performance [10]. Self-reported anxiety increased under the threatening as opposed to the safe conditions with perceived exertion estimates elevated by the duration of exercise. The results outcome demonstrated decrements in response inhibition, measured by increased false alarms, as exertion increased under the safe conditions, but improved response inhibition as exertion increased under the threatening conditions. Their findings were consistent with earlier demonstrations that anxiety induced by unpredictable threat promoted adaptive survival mechanisms, expressed through improved levels of vigilance, threat detection, cautious behavior, and harm avoidance. In a cross-sectional study that was based upon a sample of 171 middle and older Spanish workers, aged 45-65 and beyond, participants were separated, by age, between groups of older workers in mid-career (45-55 years of age) and those in their late-careers (56 years and beyond). The authors confirmed the notion that task-characteristics and, secondarily, knowledge-characteristics were the most relevant factors in perceptions of psychological work-ability among aged workers, although both age-groups displayed an marked levels of the personal mastery trait, a factor that mediates the relationships between job characteristics and both psychological leading to reinforced work-ability, sustainability subsequent job mobility intentions [11] Demographic changes modulating employment conditions have been shown to determine whether employees may enjoy job-suitability and/ or age-dependent work-ability and sustainability occupations. Thus, the relationship between work-motivation and mental health as affected by work-ability and at identifying a myriad of measures that cooperates and other businesses may apply in the construction of conglomerate age-management, work-ability policies in order to counterbalance the impact of demographic changes [12].

Finally, the recommendation of physical exercise intervention for health and well-being during leisure-time periods and ongoing, improved ability-to-work among white-collar workers presented a challenge to the sustenance of work-ability formulations. In a study of 360 white-collar Polish workers, participants who met health recommendations achieved higher scores of the work-ability index in comparison to those who were not sufficiently physically active or sedentary [13]. The outcome of a binary logistic regression indicated that fulfilling the health-related physical activity recommendations almost doubled the probability of reaching at least improved levels of work-ability. In an evaluation of the efficacy of a 12-week workplace exercise program to promote work-ability, work-performance, and patient-reported symptoms among older university employees presenting knee and/or hip osteoarthritis, marked improvements in expressions of work-ability and patient-reported outcomes, such as pain, function, depressive symptoms, were observed in the exercise group whereas no such improvements were forthcoming in the noexercise/sedentary group $[14,15]$.

\section{References}

1. Freyer M, Formazin M, Rose U (2018) Factorial Validity of the Work Ability Index Among Employees in Germany. J Occup Rehabil.

2. Chan MY, Na J, Agres PF, Savalia NK, Park DC, et al. (2018) Socioeconomic status moderates age-related differences in the brain's functional network organization and anatomy across the adult lifespan. Proc Natl Acad Sci U S A 115: E5144-E5153.

3. Sewdas R, Tamminga SJ, Boot CRL, van den Heuvel SG, de Boer AG, et al (2018) Differences in self-rated health and work ability between self-employed workers and employees: Results from a prospective cohort study in the Netherlands. PLoS One 13: e0206618.

4. Landén Ludvigsson M, Peterson G, Peolsson A (2018) The effect of three exercise approaches on health-related quality of life, and factors associated with its improvement in chronic whiplash-associated disorders: analysis of a randomized controlled trial. Qual Life Res.

5. Kadijk EA, van den Heuvel S, Ybema JF, Leijten FRM (2018) The Influence of Multi-morbidity on the Work Ability of Ageing Employees and the Role of Coping Style. J Occup Rehabil.

6. Peters E, Spanier K, Radoschewski FM, Bethge M (2018) Influence of socia support among employees on mental health and work ability-a prospective cohort study in 2013-15. Eur J Public Health 28: 819-823.

7. Jansson I, Gunnarsson AB (2018) Employers' views of the impact of mental health problems on the ability to work. Work 59: 585-598.

8. Abbasi M, Zakerian A, Akbarzade A, Dinarvand N, Ghaljahi M, et al. (2017) Investigation of the Relationship between Work Ability and Work-related Quality of Life in Nurses. Iran J Public Health 46: 1404-1412. 
9. Furu H, Sainio M, Hyvärinen HK, Kaukiainen A (2018) Work ability score of solvent-exposed workers. Int Arch Occup Environ Health 91: 559-569.

10. Cantelon JA, Giles GE, Eddy MD, Haga Z, Mahoney CR, et al. (2018) Exerting cognitive control under threat: Interactive effects of physical and emotional stress. Emotion.

11. Alcover CM, Topa G (2018) Work characteristics, motivational orientations, psychological work ability and job mobility intentions of older workers. PLoS One 13: e0195973.

12. Feißel A, Peter R, Swart E, March S (2018) Developing an Extended Mode of the Relation between Work Motivation and Health as Affected by the Work Ability as Part of a Corporate Age Management Approach. Int J Environ Res Public Health 15: pii: E779.
13. Nawrocka A, Garbaciak W, Cholewa J, Mynarski W (2018) The relationship between meeting of recommendations on physical activity for health and perceived work ability among white-collar workers. Eur J Sport Sci 18: 415-422.

14. Chopp-Hurley JN, Brenneman EC, Wiebenga EG, Bulbrook B, Keir PJ, et al. (2017) Randomized Controlled Trial Investigating the Role of Exercise in the Workplace to Improve Work Ability, Performance, and Patient-Reported Symptoms Among Older Workers With Osteoarthritis. J Occup Environ Med 59: 550-556.

15. Mulla DM, Wiebenga EG, Chopp-Hurley JN, Kaip L, Jarvis RS, et al. (2018) The Effects of Lower Extremity Strengthening Delivered in the Workplace on Physical Function and Work-Related Outcomes Among Desk-Based Workers: A Randomized Controlled Trial. J Occup Environ Med 60: 1005-1014. 